
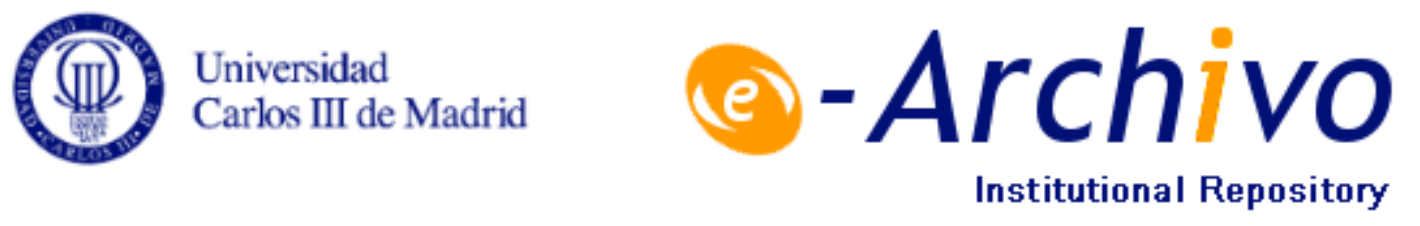

This is a postprint version of the following published document:

Rosa, T. de la, S., García-Olaya, A., Borrajo, D. (2013). A case-based approach to heuristic planning. Applied Intelligence, vol. 39, no. 1, pp. 184201. Avalaible in http://dx.doi.iorg/10.1007/s10489-012-0404-6

(C) 2013 Springer 


\title{
A case-based approach to heuristic planning
}

\author{
Tomás de la Rosa • Angel García-Olaya • Daniel Borrajo
}

\begin{abstract}
Most of the great success of heuristic search as an approach to AI Planning is due to the right design of domain-independent heuristics. Although many heuristic planners perform reasonably well, the computational cost of computing the heuristic function in every search node is very high, causing the planner to scale poorly when increasing the size of the planning tasks. For tackling this problem, planners can incorporate additional domain-dependent heuristics in order to improve their performance. Learningbased planners try to automatically acquire these domaindependent heuristics using previous solved problems. In this work, we present a case-based reasoning approach that learns abstracted state transitions that serve as domain control knowledge for improving the planning process. The recommendations from the retrieved cases are used as guidance for pruning or ordering nodes in different heuristic search algorithms applied to planning tasks. We show that the CBR guidance is appropriate for a considerable number of planning benchmarks.
\end{abstract}

Keywords Case-based reasoning - Automated planning . Search algorithms

T. de la Rosa $(\bowtie) \cdot$ A. García-Olaya $\cdot$ D. Borrajo

Departamento de Informática, Universidad Carlos III de Madrid,

Av. Universidad 30, Leganés, Madrid, Spain

e-mail: trosa@inf.uc3m.es

A. García-Olaya

e-mail: agolaya@inf.uc3m.es

D. Borrajo

e-mail: dborrajo@ia.uc3m.es

\section{Introduction}

Automated Planning is an area of the Artificia Intelligence focused on findin solutions to problems in a domainindependent way. A planning problem is a computational task whose inputs are a set of actions that can be applied in the environment, an initial state and a set of goals to be reached. The aim of Automated Planning is to fin a plan that transforms the initial state of the environment into a different state where the goals are met. Automated Planning systems have been successfully applied to real world problems such as planning space missions [21], management of fir extinctions [4], control of underwater vehicles [20] or web service compositions [18]. Heuristic search in the state space is one of the most successful approaches to solve automated planning problems. Heuristics in planning are computed in a domain-independent way, using information extracted from the domain definition the state to be evaluated, and the goal set. These heuristics usually estimate the distance between the current state and a state where goals are achieved. The estimate is used as guidance for selecting the next node in a search algorithm. Many heuristic planners integrate this basic idea. They derive heuristics from the relaxation of action negative effects such as [15] and [3]; or from causal graphs, such as [13]. Some other researchers have developed techniques for complementing or modifying these planners, such as $[5,22,26]$.

Although heuristic planners perform reasonably well in many benchmark domains, they suffer from scalability problems due to the computational cost of computing the heuristic. Indeed, these planners spend most of the planning time computing the heuristic function, since they have to obtain an estimate for every node in the search process. Usually, a single heuristic estimation can be computed in polynomial time. However, the total planning time grows exponentially 
because the number of expanded states grows exponentially too and the search algorithm needs to evaluate all of them, including non-interesting ones (i.e., nodes that do not appear in any solution plan). The problem of useless node evaluations are exacerbated in domains where the heuristic function is misleading or has poor quality. To tackle this problem different learning techniques have been used to improve the performance of planners. In fact, the community has a renewed interest for applying machine learning techniques to state-of-the-art planners. An example of this interest is the Learning Track organized in the 2008 International Planning Competition (IPC). The system CABALA [8], one of the competitors, was based on the ideas presented next.

In this article we present a learning technique that acquires domain-dependent knowledge from previously solved plans, with the aim of helping planning algorithms to avoid useless node evaluations. We have developed this technique using a Case-Based Reasoning (CBR) approach. We store the experience provided by solving previous easy problems, and then, when a new problem is being solved, we retrieve the most similar situation in order to recommend most promising nodes for the search algorithm to explore. These recommendations allow search algorithms to skip some useless node evaluations and therefore speed up the search. We also present the evaluation of this approach in eight planning benchmarks. The results show that the technique is useful for improving heuristic planners, especially when they are using greedy search algorithms.

In the following sections we describe the approach, organized as follows. The next section explains the planning model. Then, we formalize the representation used for domain-dependent knowledge. Afterward, we explain the cycle of reasoning from a CBR perspective [1]. In that section, we explain how the learned knowledge is used in different heuristic algorithms. Then, we present an experimental evaluation of the approach in eight planning benchmarks. Finally, we discuss related work and present our conclusions.

\section{Planning model}

The base planning model of this approach is the STRIPS formalism. In a domain $\mathcal{D}$, the state space $\mathcal{S}$ for a problem, and the set of actions $\mathcal{A}$ are define in terms of predicate symbols $\mathcal{Y}$, operators (action schemas) $\mathcal{O}$ and the set of object constants $\mathcal{C}$ given for a specifi task. A state $s \in \mathcal{S}$ is the set of facts (state literals) that are true in a given instant. Each fact in $s$ is an instantiation of a predicate symbol $y \in \mathcal{Y}$ in the form $y\left(c_{1}, \ldots, c_{n}\right)$ where $c_{i} \in \mathcal{C}$. An operator $o p \in \mathcal{O}$, that has $m$ parameters, can instantiate into action $a \in \mathcal{A}$ in the form $o p\left(c_{1}, \ldots, c_{m}\right)$ with $c_{i} \in \mathcal{C}$. Additionally, each action $a \in \mathcal{A}$ is define in terms of three sets of facts $(\operatorname{pre}(a), \operatorname{add}(a), \operatorname{del}(a))$, where pre $(a)$ are the preconditions that should be true for the action to become applicable, $\operatorname{add}(a)$ are the facts that become true when the action is executed, and $\operatorname{del}(a)$ are the facts that are no longer true after the action execution. If an action $a$ is applied in a state $s$, denoted by $\operatorname{apply}(a, s)$, the resulting state will be $s^{\prime}=(s-\operatorname{del}(a)) \cup \operatorname{add}(a)$.

A planning task in $\mathcal{D}$ is define as the tuple $(\mathcal{C}, I, G)$, where $\mathcal{C}$ is the set of constants for instantiating $\mathcal{S}$ and $\mathcal{A}, I \in \mathcal{S}$ is the initial state and $G$ is the set of facts (a partially define state) that represent the goals. Solving a planning task implies findin a plan $\pi=\left\{a_{1}, \ldots, a_{n}\right\}$ such that, if applied consecutively starting from the initial state, it leads to a state where all the goals are true, i.e., $\mathcal{G} \subseteq \operatorname{apply}\left(a_{n}, \operatorname{apply}\left(a_{n-1}, \ldots, \operatorname{apply}\left(a_{1}, \mathcal{I}\right)\right)\right)$.

Moreover, we defin additional functions to handle the case base representation used later, namely:

$-\operatorname{pred}(l)=y$ is the predicate symbol of the fact $l$

$-\arg (l)=\left(c_{1}, \ldots, c_{n}\right)$ are the arguments of the fact $l$

- $\operatorname{opt}(a)=o p$ is the operator of the action $a$

$-\operatorname{argop}(a)=\left(c_{1}, \ldots, c_{m}\right)$ are the parameters of the action $a$

Some parts of our model depend on features from the relaxed plan heuristic [15]. In general, heuristics based on delete relaxation compute their estimation by considering a relaxed version of the planning task, which is the same planning task, but using a variation of the action set $\mathcal{A}$ (i.e., it does not contain the $\operatorname{del}(a)$ set). In particular, the aim of the relaxed plan heuristic is findin a relaxed plan $\pi^{+}$that solves the relaxed planning task. The relaxed plan is extracted from a Relaxed Planning Graph (RPG). An RPG is a sequence of proposition and action layers $P_{0}, A_{0}, P_{1}, A_{1}, \ldots, P_{n-1}, A_{n}, P_{n}$. The firs proposition layer, $P_{0}$, contains the propositions in the state to be evaluated, $s$. Then, each action layer contains all applicable actions given the previous proposition layer. The positive effects of all actions in a layer are included in the next proposition layer. Thus, each proposition layer contains the set of achieved propositions so far. Hoffmann and Nebel [15] showed that a relaxed plan can be extracted from an RPG in polynomial time. The relaxed plan extraction algorithm selects in each proposition layer the facts marked as goals and then selects actions from the previous layer that achieve the goals, including as new goals the preconditions of the selected actions. This algorithm corresponds to a backtracking-free version of the one used in the GRAPHPLAN planner [2]. Hoffmann and Nebel [15] also introduced the pruning strategy of the helpful actions. A helpful action is an applicable action (i.e., it belongs to the $\mathrm{f}$ rst action layer in RPG) that achieves a fact marked as a goal (or sub-goal) during relaxed plan extraction. For an extended description of heuristic planning refer to [12]. 


\section{Definition}

A typed sequence is the basic piece of knowledge used by our CBR algorithm. We present a set of definition first

Definitio 1 Given a state $s$, an object sub-state is the set of state literals in which the object appears as an argument. The sub-state for the object $o$ in the state $s$ is denoted by

$s_{o}=\{l \mid l \in s \wedge o \in \arg (l)\}$

For instance, suppose that in the Blocksworld domain we have a state $s=\{($ on $A B)$ (ontable B) (clear A) (holding $C)\}$. We say that the object sub-state for block $A$ is $\{($ on A B) $(\operatorname{clear} A)\}$.

Definitio 2 A property of an object $o$ in a literal $l$ is the position of $o$ in $l$. It will be represented as a predicate symbol subscripted by a number representing the argument position in the state literal.

$U_{o}(l)=\operatorname{pred}(l)_{i} \mid \arg (l)=\left(c_{1}, \ldots, c_{n}\right) \wedge o=c_{i}$

For instance, $o n_{1}$ is the property define for the block $A$ in the literal (on $A B$ ), and $o n_{2}$ is the property for the block $B$. Therefore, a predicate of arity $n$ produces $n$ properties. This concept was originally introduced by [11], where properties are used to derive domain types.

Since properties represent object positions in state literals and each position has a particular domain type, we can use properties for referring to objects in an abstract way. Thus, we can transform an object sub-state into what we will call a typed sub-state, using the properties for the literals that belong to an object sub-state. Since two or more literals can produce the same property for an object, we use the concept of collection as a generalization of the concept of sets. A collection is an unordered group of elements where a particular element can appear more than once.

Definitio 3 A typed sub-state is the collection of properties that abstracts an object sub-state. We will represent the typed sub-state for an object $o$ in the state $s$ as:

$\varphi_{s, o}=\left\{U_{o}(l) \mid l \in s_{o}\right\}$

In the previous example, the object sub-state for block $A$ is transformed into the typed sub-state $\left(\right.$ on $_{1}$ clear $\left._{1}\right)$. If we apply the action (STACK C A) to the state in the example, the new object sub-state for block $A$ is [(on $A B)($ on $C A)]$. Therefore, the new typed sub-state becomes $\left(o n_{1} o n_{2}\right)$.

Since objects of a particular planning instance are not relevant when recognizing generalized domain transitions, we need to represent typed sub-states that abstract any valid object sub-state in the domain.
Definitio 4 An arbitrary typed sub-state $\mathcal{T}$ is a property collection that abstracts all possible object sub-states $s_{O}$ that produce equal $\varphi_{s, o}$, for an arbitrary object $o$.

For instance, the arbitrary typed sub-state $\mathcal{T}=\left(o_{1}\right.$ clear $_{1}$ ) represents all states where any block is on another block and clear. The typed sub-state $\varphi_{s, o}$ is related to an object $o$, but $T$ is related to all objects having the same typed sub-state.

In a domain, we can identify common transitions of a given object and associate them with the applied action, assuming that the object is a parameter of the action. Thus,

Definitio 5 A typed sub-state transition is a structure of the form

$\left\langle\mathcal{T}\right.$, op, $\left.\mathcal{T}^{\prime}\right\rangle$

representing an observed transition generated when a typed sub-state $w_{o, s}$ is transformed into $w_{o, s^{\prime}}$ after applying an action $a$ such that $o p=\operatorname{opt}(a)$. The action $a$ is generalized to its operator $o p$ since the other parameters are not relevant for the object perspective of the representation.

Definitio 6 A typed sequence $\mathcal{Q}$ is a sequence of ordered pairs of the form

$\mathcal{Q}=\left\{\left(\mathcal{T}_{0}, \emptyset\right),\left(\mathcal{T}_{1}, o p_{1}\right), \ldots,\left(\mathcal{T}_{n}, o p_{n}\right)\right\}$

that represents an object-centered abstraction of a plan, in which each pair is an arbitrary typed sub-state with the operator used to reach that typed sub-state.

A typed sequence is a way of summarizing the typed sub-state transitions. For an object $o$ and a plan $\pi=$ $\left\{a_{1}, a_{2}, \ldots, a_{n}\right\}$, we can generate a typed sequence where $\mathcal{T}_{0}=\varphi_{I, o}$ is the typed sub-state for the initial state, and each $\mathcal{T}_{i}$ is the typed sub-state obtained from the state $s$ reached when the action $a_{i}$ is applied.

Since typed sequences focuses on particular objects, not all actions in a solution plan may affect that object. Thus, we defin a void operator (no-op) that substitutes the real one in case the object is not a parameter of the corresponding applied action. Therefore, in any sequence step $\left(\mathcal{T}_{i}\right.$, no-op $)$, we assume that $\mathcal{T}_{i}=\mathcal{T}_{i-1}$ and the action $a_{i}$ is not relevant for the object generating the sequence. Additionally, we use an integer, together with the no-op, to indicate the number of times that irrelevant actions have been applied. This allows us to maintain a compact representation of typed sequences. Any action producing a no-op at the end of a sequence is not taken into account, since the rest of the plan has no relation with the particular object and there is no need to collect any additional transitions for the object.

Figure 1 shows a complete typed sequence for an object (block $A$ ) of type Block in the Blocksworld domain. The 
firs pair in the sequence has the typed sub-state corresponding to the initial state and it has no associated action. The other pairs are generated as explained before. The action ( PICKUP C) produces a no-op action, because it does not affect block $A$ and the typed sub-state remains the same.

Definitio 7 Given a relaxed plan $\pi^{+}=\left\{a_{1}, \ldots, a_{n}\right\}$, the relaxed plan footprint for an object $o$ is the sequence of pairs $\left\{\left(\mathcal{O}_{1, o}, \mathcal{T}_{1}\right), \ldots,\left(\mathcal{O}_{n, o}, \mathcal{T}_{n}\right)\right\}$ where $\mathcal{O}_{i, o}$ represents the set of operators that affect object $o$ in the layer $i-1$ of the RPG and add some properties for the collection $\mathcal{T}_{i}$ in layer $i$. It is computed as

$\mathcal{O}_{i, o}=\left\{\operatorname{opt}(a) \mid a \in \pi^{+} \wedge a \in A_{i-1} \wedge o \in \operatorname{argop}(a)\right\}$

Each $\mathcal{T}_{i}=\varphi_{s_{i}{ }^{+}, o}$ is the collection of added properties where $s_{i}^{+}=\left\{\operatorname{add}(a) \mid a \in \pi^{+} \wedge a \in A_{i-1}\right\}$.

The relaxed plan footprint is a compact way of partially representing a typed sequence. Thereby, the footprint can serve as a retrieval key, since it can be computed at the beginning of the planning process (i.e., after computing the heuristic estimation of the initial state). The relaxed plan footprint has two main differences with a normal typed sequence. First, it only keeps track of added properties through

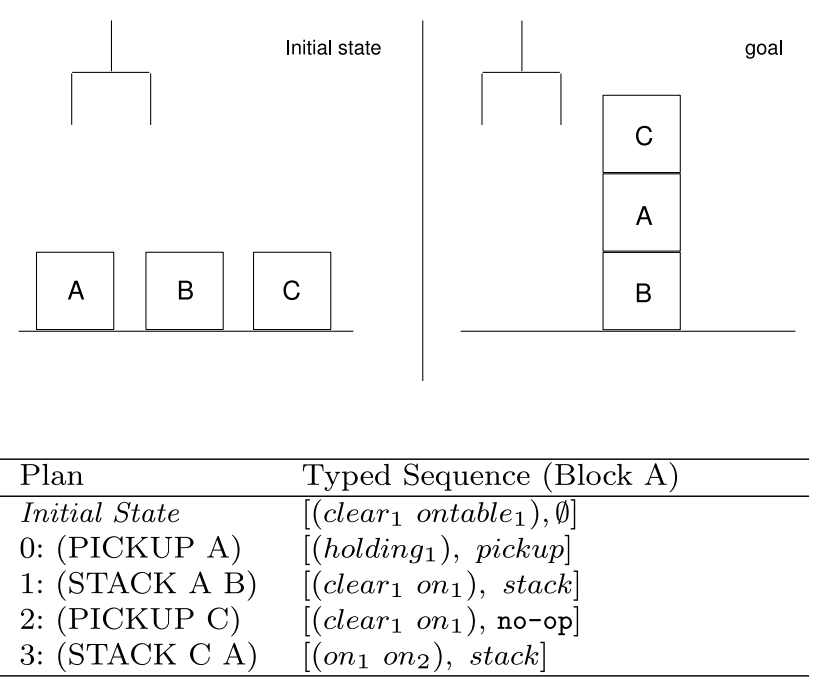

Fig. 1 An example of a typed sequence in the Blocksworld domain consecutive RPG layers in the relaxed plan of the initial state. Second, it stores the associated operators by layers. Therefore, there is no precedence relation among them. Figure 2 shows the computation of the relaxed plan footprint for blocks $A, B$ and $C$ of the example in Fig. 1. The relaxed plan for the initial state coincides with the real plan, even though the relaxed plan footprint differs from the typed sequence generated for the example.

\section{CBR cycle}

In this section we explain how typed sequences are used in a complete CBR cycle [1]. This cycle comprises retaining, retrieving, reusing and revising the cases of a particular domain. The learning system has been developed as a trainer/advisor module that supports a heuristic planner during the search process, as shown in Fig. 3. This system was built within SAYPHI, a planning and learning framework in which different learning techniques can be integrated with a common planner $[7,8]$.

The system fow is as follows. A set of training problems is solved by the heuristic planner, and the solutions are used to generate typed sequences and populate the case base (i.e., the "Retain" process in Fig. 3). In order to solve new problems, modifie versions of the heuristic search algorithms request similar sequences that match the initial state, goals and relaxed plan footprint of the new problem (i.e., the "Retrieval" process). The retrieved sequences are used to recommend node ordering or pruning according to the states that reproduce the same transitions encoded in the sequences (i.e., the "Reuse" process). Finally, new solutions and an evaluation score are used to recognize which types are useful in the case base and update it accordingly (i.e., the "Revision" process). Next, we give an explanation of each phase.

\subsection{Retain}

This process manages the generation and maintenance of the case base. Its main tasks consist of (a) the generation of typed sequences from a set of training problems, (b) the case storage in the case base structure and (c) the maintenance,
Fig. 2 An example of how to compute the relaxed plan footprint

\begin{tabular}{lllll}
\hline & $A_{0} \in \pi^{+}$ & $s_{1}^{+}$ & $A_{1} \in \pi^{+}$ & $s_{2}^{+}$ \\
\hline Relaxed Plan & (PICKUP A) & $($ holding $A)$ & $($ STACK A B) & $\begin{array}{l}(\text { on A B }) \\
\text { clear A }) \\
(\text { on } C \text { A }) \\
\text { clear } C)\end{array}$ \\
\hline Footprint A & PICKUP & holding $_{1}$ & STACK,STACK & on $_{1}$, clear $_{1}$, on $_{2}$ \\
\hline Footprint B & $\emptyset$ & $\emptyset$ & STACK & on $_{2}$ \\
\hline Footprint C & PICKUP & holding $_{1}$ & STACK & on $_{1}$, clear $_{1}$ \\
\hline
\end{tabular}


Fig. 3 SAYPHI: a heuristic planner supported by a CBR system

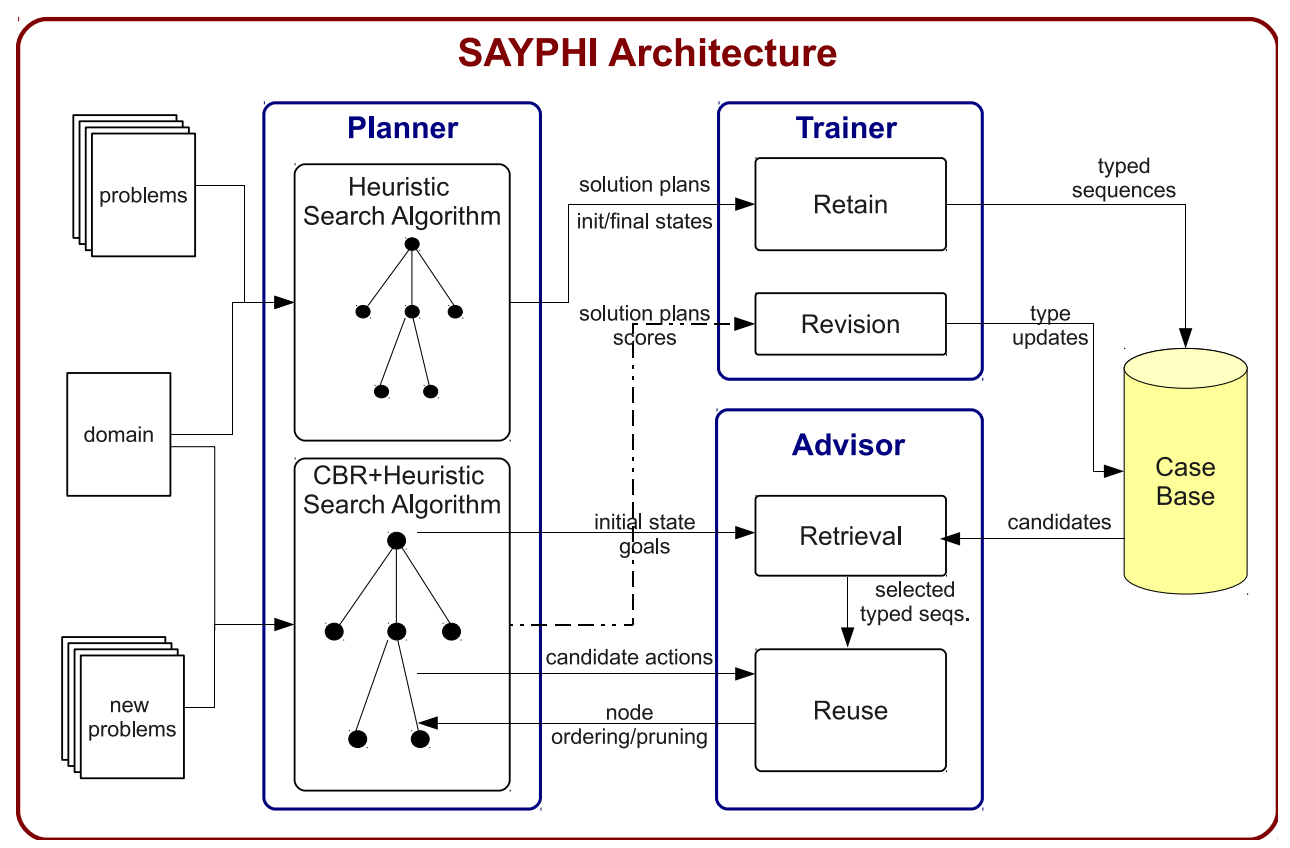

which implies merging repeated sequences, deleting useless ones, etc. From a more general point of view, all these tasks represent the training phase of the learning system.

\subsubsection{Storage}

This task stores the generated sequences in domain-dependent structures for later use. A case for a particular domain $\mathcal{D}$ is a tuple $\{i, \mathcal{F}, \mathcal{Q}\}$, where $i$ is a case number, $\mathcal{F}$ is the relaxed plan footprint, and $\mathcal{Q}$ is the typed sequence. The relaxed plan footprint is computed after evaluating the initial state with the heuristic function of the relaxed plan. Relaxed plan footprints collect information that can help to recognize similar sequences that differ from each other in their middle steps.

\subsubsection{Generalization}

Once cases have been generated, a generalization process tries to merge new sequences with previous ones. For this purpose, we defin the concept of equal and equivalent sequences.

Definitio 8 Two typed sequences are equal if all their ordered pairs $\left(\mathcal{T}_{i}, o p_{i}\right)$ are equal.

Definitio 9 Two typed sequences are equivalent if when removing in both sequences all pairs $\left(\mathcal{T}_{i}, o p_{i}\right)$ such that $o p_{i}=n o-o p$, the resulting sequences are equal.

Thus, all pairs of equal or equivalent sequences are merged in order to generate a new sequence. Figure 4

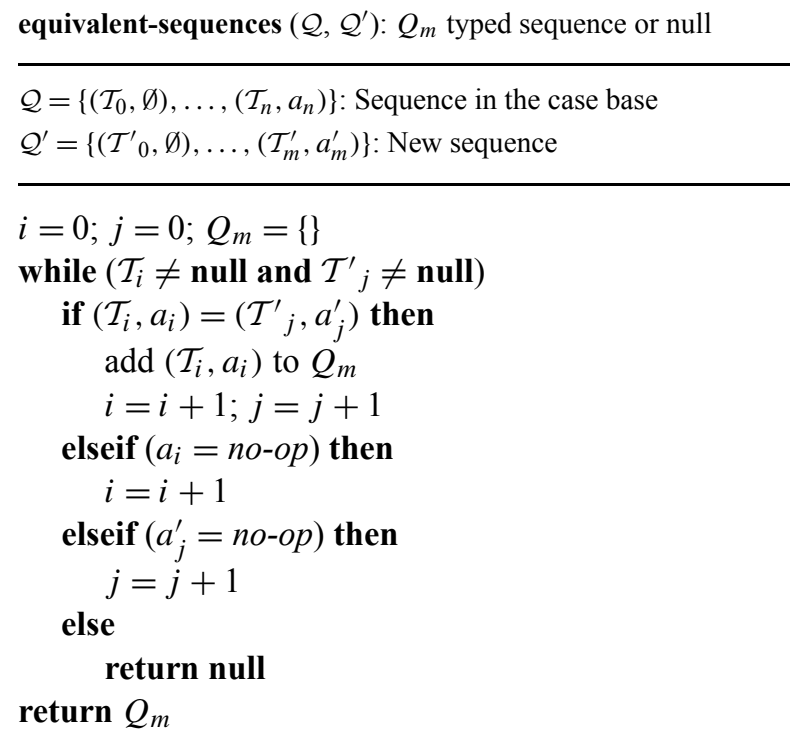

Fig. 4 The algorithm for merging two sequences

shows the algorithm for the merging process. If the function equivalent-sequences returns a new sequence, it is stored in the case base and the input sequences are removed. If the new sequence is completely new (i.e., function equivalentsequences returns null), it is stored as is in the case base.

\subsection{Retrieval}

Given a new planning task $(\mathcal{C}, I, G)$ for the domain $\mathcal{D}$, the retrieval process searches the case base for the most similar cases to this task. Given that each planning state contains information on several objects, we retrieve one case for each 
object in the planning task. For each object $o$ in $C$, the features used by the similarity function are:

- the object type of $o$

- the typed sub-state generated from the initial state: $\varphi_{I, o}$

- the typed sub-state generated from the set of goals: $\varphi_{G, o}$

- the relaxed plan footprint computed for the initial state: $\left\{\left(\mathcal{O}_{1, o}, \varphi_{s_{1}^{+}, o}\right), \ldots,\left(\mathcal{O}_{n, o}, \varphi_{s_{n}^{+}, o}\right)\right\}$

The object type is used to pre-select the subset of cases corresponding to its type, so it is not directly used to compute the similarity metric.

\subsubsection{Similarity metric}

The similarity metric used for typed sub-state comparisons takes into account that the number of properties of an object can vary depending on the size of the planning instance. Accordingly, we defin three levels or degrees of matching to indicate how similar two typed sub-states are. We represent typed sub-states $\mathcal{T}$ as pairs $(X, m)$, where $X$ is the set of non repeated properties in $\mathcal{T}$, and $m(x)$ is the function representing the number of times the element $x \in X$ appears in $\mathcal{T}$. For instance, in the Logistics domain, if the object sub-state for the truck $\operatorname{Tr} 1$ is $s=\{($ at Tr1 Locl) (in Pkgl Trl) (in Pkg2 $\operatorname{Tr} 1)\}$ the corresponding typed sub-state is $\left(a t_{1} i n_{2} i n_{2}\right)$. This typed sub-state can be written as $\left(\left(a t_{1} i n_{2}\right),\left(\begin{array}{ll}1 & 2\end{array}\right)\right)$ under this representation.

Definitio 10 Given two typed sub-states $\mathcal{T}_{1}$ and $\mathcal{T}_{2}$, expressed as pairs in the form $\left(X_{1}, m_{1}\right)$ and $\left(X_{2}, m_{2}\right)$, we defin the matching level as follows:

1. There is a total match of $\mathcal{T}_{1}$ with $\mathcal{T}_{2}$, represented as $\mathcal{T}_{1} \subseteq \mathcal{T}_{2}$, when all properties in $\mathcal{T}_{1}$ are present in the same amount in $\mathcal{T}_{2}$. Formally, this relation is define as

$$
\mathcal{T}_{1} \subseteq \mathcal{T}_{2} \leftrightarrow X_{1} \subseteq X_{2} \wedge \forall x \in X_{1} m_{1}(x)=m_{2}(x)
$$

2. The relation $\mathcal{T}_{1} \varsubsetneqq \mathcal{T}_{2}$ represents a partial matching of $\mathcal{T}_{1}$ with $\mathcal{T}_{2}$, when all properties in $\mathcal{T}_{1}$ are present in $\mathcal{T}_{2}$, but not all of them appear the same number of times. Formally, this relation is define as

$$
\mathcal{T}_{1} \varsubsetneqq \mathcal{T}_{2} \leftrightarrow X_{1} \subseteq X_{2} \wedge \exists x \in X_{1} m_{1}(x) \neq m_{2}(x)
$$

3. The relation $\mathcal{T}_{1} \nsubseteq T_{2}$ represents that $\mathcal{T}_{1}$ has no matching with $\mathcal{T}_{2}$ because not all properties in $\mathcal{T}_{1}$ are in $\mathcal{T}_{2}$. Formally, this relation is define as

$$
\mathcal{T}_{1} \nsubseteq \mathcal{T}_{2} \leftrightarrow X_{1} \nsubseteq X_{2}
$$

These relations are not commutative (i.e., $\mathcal{T}_{1} \subseteq \mathcal{T}_{2}$ does not imply $\mathcal{T}_{2} \subseteq \mathcal{T}_{1}$ ). A typed sub-state generated for an object in the new problem should be equal to the $\mathrm{f} \mathrm{rst/last} \mathrm{step}$ in the stored sequence, or a subset of this step, but not the opposite. This is because typed sub-state of goals could be partially define (goals are partially define states), but the last steps of a stored sequence have the complete typed sub-state. For instance, in the Blocksworld domain, the last step in the sequence generated for block $B$ in Fig. 1 is [ $\left(\right.$ on $_{2}$ ontable $\left._{1}\right)$, stack]. However, if a block in a new problem does not need to be on another block in the goals, the goal typed sub-state is $\left(o n_{2}\right)$ if the on-table predicate is not specifie as a goal, which normally occurs. Therefore, considering the define matching levels for the retrieval, $\mathcal{T}_{1}$ will refer to a typed substate generated from an object in the new problem, and $\mathcal{T}_{2}$ will refer to a typed sequence in a stored sequence.

Relations in Definitio 10 can be extended to relaxed plan footprints. Since relaxed plan footprints keep properties and operators by layers, in the extension it is relevant to take empty layers into account.

Definitio 11 Two relaxed plan footprints have a total match if each pair $\left(\mathcal{O}_{i, o}, \mathcal{T}_{i}\right)$ is equal in both footprints. Two relaxed plan footprints have a partial match when they have a total or a partial match for each pair $\left(\mathcal{O}_{i, o}, \mathcal{T}_{i}\right)$, after removing in both sequences pairs such that $\mathcal{O}_{i, o}=\emptyset \wedge \mathcal{T}_{i}=\emptyset$.

The similarity metric is define in terms of the matching level for the initial and goal sub-states. The matching level for the relaxed-plan footprints is used to break ties if two cases obtain the same value for the similarity metric with the new case. A tie in the similarity metric normally occurs when two sequences have the same initial and goal typed sub-states. Then, the tie needs to be broken taking into account the intermediate steps, partially abstracted by the relaxed plan footprint. Given an object $o$ of the new planning task $(\mathcal{C}, I, G)$, the similarity metric is computed with Eq. (3).

$\mathcal{M}\left(o, \mathcal{Q}_{i}\right)=2 W\left(\varphi_{I, o}, \mathcal{T}_{0}\right)+W\left(\varphi_{G, o}, \mathcal{T}_{n}\right)$

where $\mathcal{Q}_{i}$ is the sequence $i$ in the case base of the same type of $o$. The function $W$ returns a numerical value for distinguishing relations described in Definitio 10. The match for the initial typed sub-states is more relevant in the equation because goal typed sub-states are partially defined Moreover, in any given planning task this value is 0 for many objects (i.e., the object does not appear in any goal). For simplicity we use the following equivalence for the matching relations.

$W\left(\mathcal{T}_{1}, \mathcal{T}_{2}\right)= \begin{cases}2 & \text { if } \mathcal{T}_{1} \subseteq \mathcal{T}_{2} \\ 1 & \text { if } \mathcal{T}_{1} \varsubsetneqq \mathcal{T}_{2} \\ 0 & \text { if } \mathcal{T}_{1} \nsubseteq \mathcal{T}_{2}\end{cases}$

If there is a tie in the similarity metric, the function $W$ is applied to the footprints of each sequence, and the one with greater value is selected. 


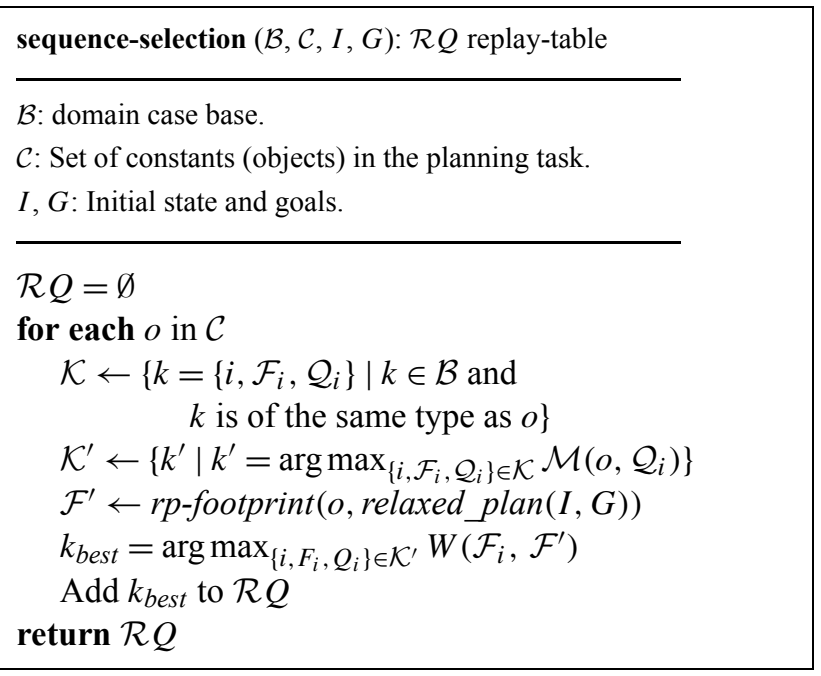

Fig. 5 Algorithm for ranking and selecting typed sequences for a new problem

\subsubsection{Ranking and selection}

The goal of the retrieval process is to build the replay table. It is define as the set of pairs $\left\langle\mathcal{T}_{i}, o_{i}\right\rangle$, where $\mathcal{T}_{i}$ are the retrieved sequences, and $o_{i}$ is the object it was retrieved for. Figure 5 shows the pseudo-code for building the replay table. For each object in the new problem, a subset of sequences maximizing the similarity metric is selected. Then, from this subset, the sequence with the best match in the relaxed plan footprint is added to the replay table as the retrieved sequence for the object. The function $r p$-footprint computes the relaxed plan footprint of the initial state for the object $o$ according to Definitio 7 .

\subsection{Reuse}

The idea of reusing the knowledge kept in the typed sequence consists of replaying the typed sub-state transitions during the forward state search. The retrieved sequences are managed through the replay table, which keeps track of the current step being followed for each sequence, as in previous work on case-based planning [25]. The advice given by typed sequences is usually independent of the search algorithm. Accordingly, we use the concept of a "recommended node" to recognize a promising successor suggested by the CBR component; that is, it reproduces a sub-state transition for an object in the replay table. We denote the replay table with the symbol $\mathcal{R} Q$ and the function next_step $(\mathcal{R} Q, o$ ) returns the next pair $\left(\mathcal{T}_{i}, o p_{i}\right)$ of the sequence retrieved for object $o$. Given a state $s$, the successors are represented as pairs $\left(s^{\prime}, a\right)$, where $s^{\prime}=\operatorname{apply}(a, s)$. In order to recognize whether a successor replays the current transition in the re- trieved sequence for an object $o$, we defin a function:

$$
\Gamma\left(\mathcal{R} Q, o, s^{\prime}, a\right)=\left\{\begin{array}{cc}
1 & \text { If } n \text { next_step }(\mathcal{R} Q, o)=\left(\mathcal{T}_{i}, o p_{i}\right) \\
& \wedge o \in \operatorname{argop}(a) \wedge \text { opt }(a)=o p_{i} \\
& \wedge W\left(\varphi_{s^{\prime}, o}, \mathcal{T}_{i}\right)>0 \\
0 \quad \text { otherwise }
\end{array}\right.
$$

Definitio 12 A recommended node is a candidate node that reproduces the typed sub-state transition in the sequence retrieved for a parameter (object) of the applied action in the node. Formally, a successor $\left(s^{\prime}, a\right)$ is a recommended node if:

$\exists o \in \operatorname{argop}(a) \mid \Gamma\left(\mathcal{R} Q, o, s^{\prime}, a\right)=1$

Definitio 13 The recommendation ratio for a successor is the level of recommendation of a node related to the total number of action parameters of that node. Formally, the recommendation ratio for a successor $\left(s^{\prime}, a\right)$ is define with the function

$r_{c b r}\left(\mathcal{R} Q, s^{\prime}, a\right)=\frac{\sum_{\forall o \in \operatorname{argop}(a)} \Gamma\left(\mathcal{R} Q, o, s^{\prime}, a\right)}{|\operatorname{argop}(a)|}$

The recommended ratio is used for ranking successors in search algorithms, with the aim of prioritizing those nodes that replay the most sequences.

\subsection{Advising heuristic search}

The control knowledge given by the typed sequences can be integrated within any heuristic search algorithm. There are two basic strategies of using retrieved sequences:

- Pruning Strategy: a recommended node (if exists) can be directly selected, discarding other successors. This acts similar to a state-action policy.

- Ordering Strategy: a recommended node can be preferred for evaluation in a greedy algorithm that distinguishes between node generation and node evaluation.

In the following sections we present how these strategies can be integrated into three search algorithms commonly used in planning.

\subsubsection{CBR hill-climbing}

The f rst straightforward application of the pruning strategy consists of directly selecting a recommended node whenever advice is available among the current state successors. For instance, after a node expansion in the hill-climbing algorithm, if a successor is recommended, it is selected and no other evaluations are performed. If there is no advice, all nodes are evaluated and the one with the best heuristic estimation is selected. Figure 6 shows the complete pseudocode for this algorithm. 
Figure 7 shows an example of how typed sequences are replayed in CBR hill-climbing when solving a problem in the Blocksworld domain. The top of the illustration shows

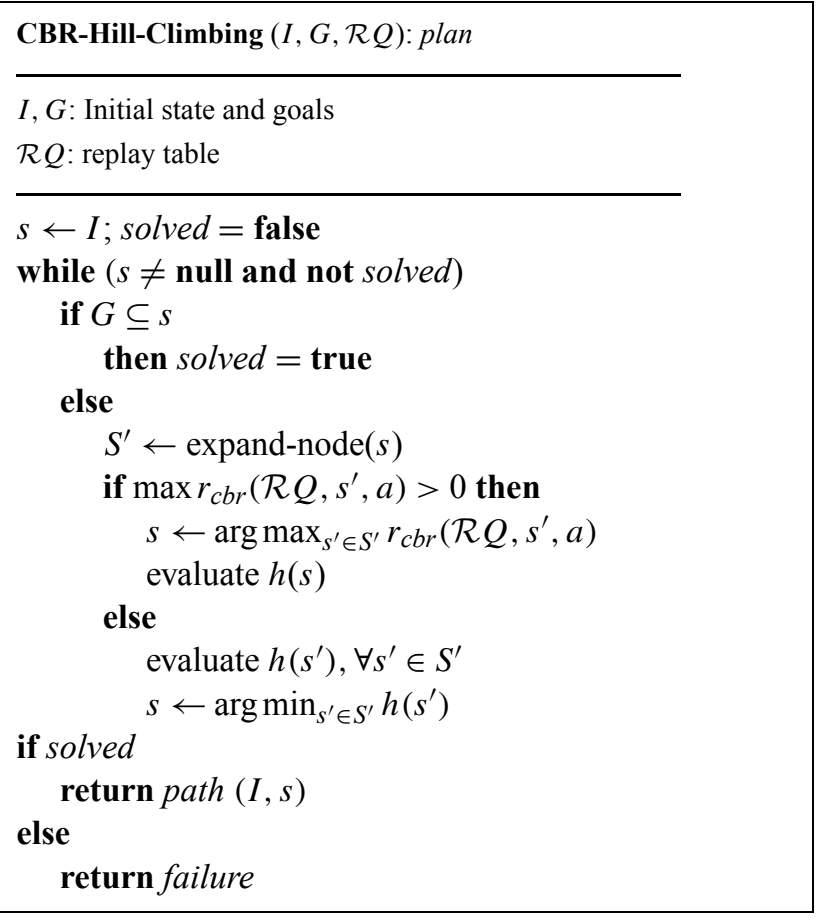

Fig. 6 A hill-climbing algorithm guided by CBR recommendations the configuratio of the initial and fina state. At the bottom, there is a plan that solves the problem along with the four retrieved sequences, one for each block in the problem. In this domain, all objects are of type Block. Next, we explain how the replay occurs. The search tree in this algorithm only considers helpful actions. The search can also be followed using the search tree in Fig. 8.

1. The only two successors of the initial state, (UNSTACK A B) and (UNSTACK C D), have both recommended ratio 1, because their two arguments match the transitions in their respective sequence. Both actions are correct for findin an optimal plan. The search selects the action (UNSTACK A B) since it appears frst after the node expansion.

2. Action (PUTDOWN A) matches the third step in the sequence retrieved for block $A$. It has ratio 1 . The action (STACK A C) has ratio 0, because it does not match the transition for block $A$ or block $C$.

3. Actions (PICKUP B) and (UNSTACK C D) have ratio 1. The second one is preferred because in this action two sequences are being followed (i.e., sequences for block $C$ and $D$ ). When a tie in $r$ occurs, it is solved preferring the action that replays more sequences.

4. Action (PUTDOWN $C$ ) is selected because it has ratio 1 . (STACK B C) has ratio 0. The action (STACK C A) has ratio $\frac{1}{2}$ because block $C$ does not match, but the current transition of block $A$ does, since it is expecting
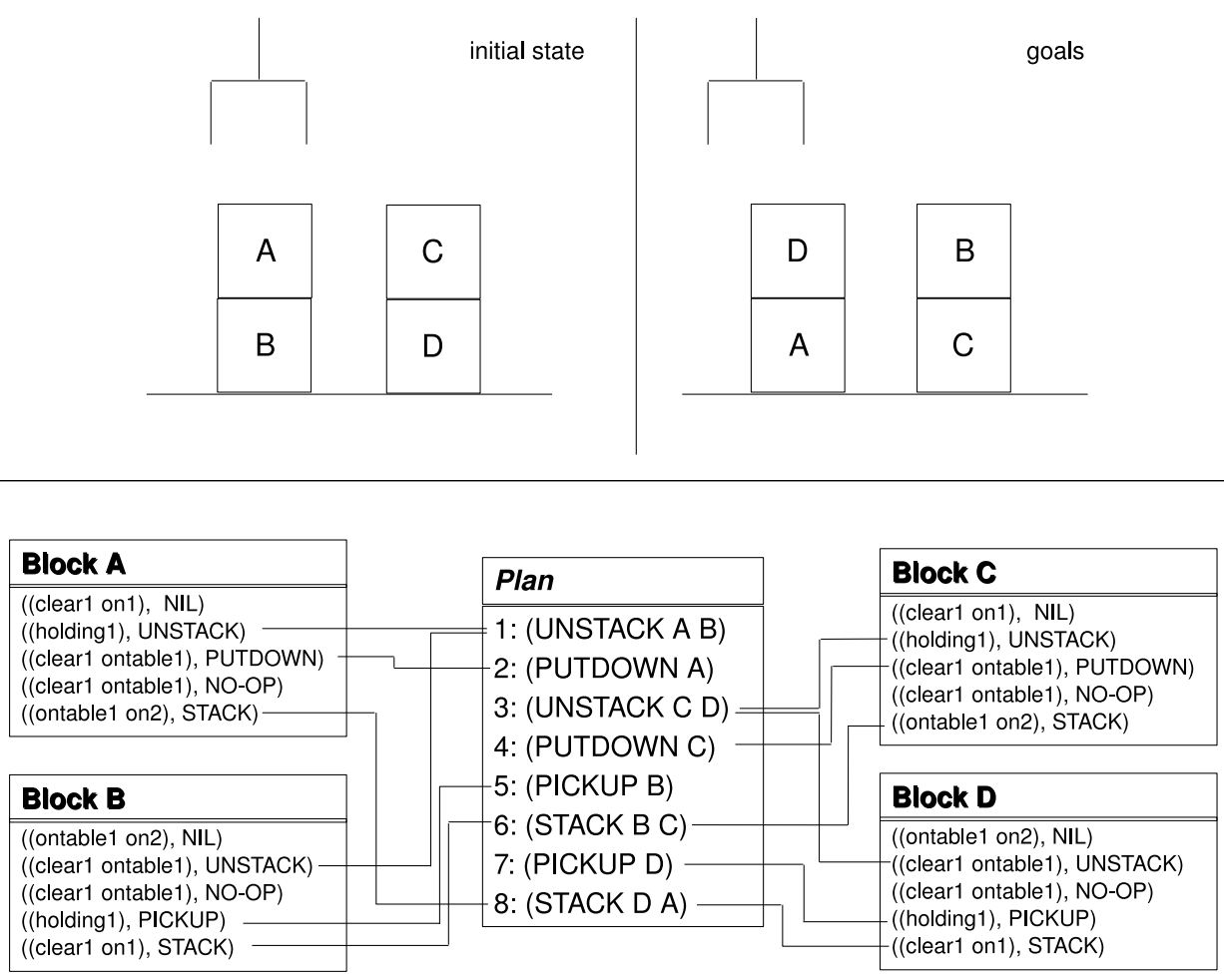

Fig. 7 Example of replaying typed sequences in the CBR Hill-Climbing algorithm 


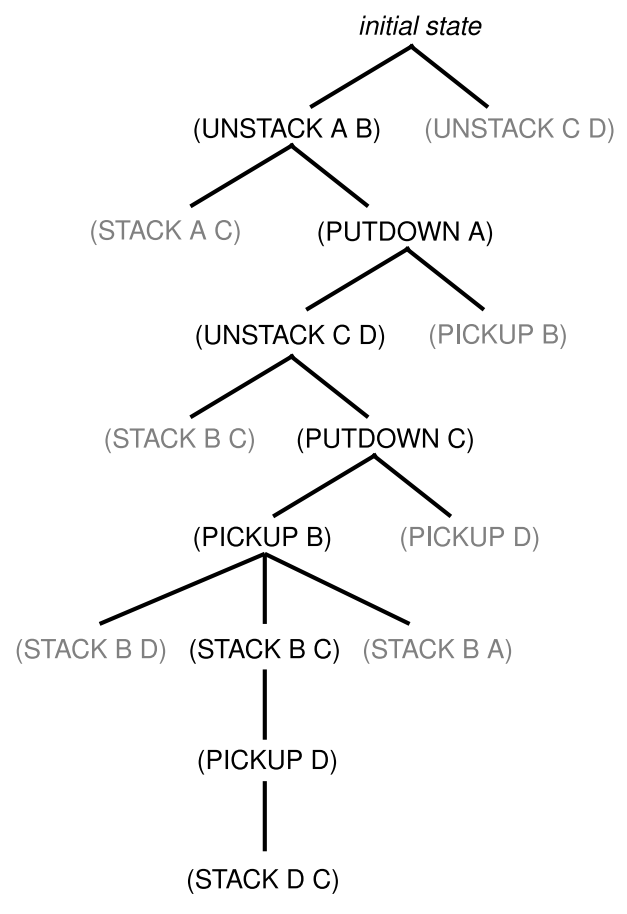

Fig. 8 The search tree resulting for the replay process of the example in Fig. 7. Nodes in gray are helpful actions not selected by the recommendations

to have a block on it in the transition $\left\{\left(\right.\right.$ ontable $_{1}$ on $\left._{2}\right)$, stack\}.

5. Actions (PICKUP B) and (PICKUP D) have ratio 1. In this situation we can clearly appreciate how the pruning of the helpful actions technique chooses a small and coherent set of successors (i.e., picking up block $A$ or $C$ are not considered as successors). Both actions in the helpful action set are correct. Action (PICKUP B) is selected because of the expansion order.

6. Action (STACK B D) has ratio $\frac{1}{2}$, because block $B$ matches, but block $D$ does not. Actions (STACK B C) and (STACK B A) have ratio 1. In this situation, the right choice is selected by chance (i.e., it appears $\mathrm{frst}$ in the successors). Action (STACK B A), even with ratio 1 , could produce a wrong choice in the search. This action appears as helpful because it reaches the sub-goal of having the robot arm empty, which is needed later.

7. Action (PICKUP D) with ratio 1 is the only successor.

8. Action (STACK D C) with ratio 1 is the only successor. With this action the problem goals are reached.

\subsubsection{CBR EHC}

This algorithm modifie the Enforced Hill-Climbing algorithm (EHC) [15] to take advantage of the greedy feature in terms of node evaluations. EHC performs a breadth-firs search from a node $s$ until it find a node $s^{\prime}$ that has a better heuristic value. This process repeats iteratively until findin the goals or running out of successors. States in the same breadth level are evaluated in the order given by the parent node expansion. This means that if in a certain breadth level, a state improving the heuristic estimation is evaluated first the rest of evaluations can be avoided. The modificatio for EHC that integrates CBR recommendations affects the evaluation node ordering. After a node expansion, each successor is evaluated only if it is a recommended node. Otherwise, the evaluation is postponed and the node is placed into a delayed list. If none of the recommended nodes improves the current heuristic estimation, the rest of successors are evaluated and the algorithm continues normally.

This modificatio to EHC seems interesting because it keeps the same layout for the algorithm, giving the opportunity of avoiding useless node evaluations. The main drawback is that CBR-EHC still relies upon the heuristic function. Therefore, the domain topology and the existence of different types of plateaus [14] will affect the fina performance of this algorithm. Suppose that EHC needs to expand the breadth-firs search until a certain depth $d$ in order to escape a plateau. If there is only one node at depth $d$ that improves the heuristic estimation, the evaluation of this node (if marked as recommended) can avoid the rest of evaluations. On the other hand, if all nodes at depth $d$ are plateau exits, matching states for findin a CBR advice becomes a waste of time. Accordingly, only an experimental evaluation can determine the benefi of this technique in certain domains.

\subsubsection{CBR WBFS}

The Weighted Best-First Search (WBFS) is another algorithm commonly used in forward heuristic planning. The CBR version tries to use the typed sequence recommendations to break ties in the evaluation function $f(n)=g(n)+$ $w_{h} \times h(n)$. In order to keep the same layout for the algorithm, we just modify the evaluation function with the formula:

$f(n)=g(n)+w_{h} \times\left(h(n)+1-h_{c b r}(n)\right)$

where $h_{c b r}(n)$ returns the recommended ratio computed for the successor $\left(s^{\prime}, a\right)$ in $n$. The idea consists of modifying the value that $h(n)$ contributes to the standard formula. Thereby, a recommended node (if any) will be preferred to continue the search. The new evaluation function is only useful when $w_{h}>1$ because otherwise the algorithm will not take advantage of the new ordering imposed to $f(n)$. Let us consider an example with an admissible heuristic. If $s_{g}$ is a goal state, all generated nodes that hold $f(n)<g\left(s_{g}\right)$ should be evaluated before stopping the search, therefore it does not make sense to order the evaluation of these nodes. Although the relaxed plan heuristic is non-admissible, some experimental evaluations have shown a similar effect. Nevertheless, WBFS with 
$w_{h}>1$ has the effect (which increases with bigger $w_{h}$ ) of not reconsidering specifi nodes while it is descending in the search tree. This means that the node ordering is relevant and preferring more promising nodes could affect the fina performance.

CBR-WBFS has some differences with greedy algorithms as CBR-Hill-Climbing or CBR-EHC. In greedy search algorithms, a single replay table can keep track of the current step of sequences. However, in CBR-WBFS, nodes being expanded can belong to different paths, so it is necessary to keep track of the current step of the replay table followed in each path. Therefore, in WBFS-CBR, each node holds a set of references indicating the current step being followed at that node.

\subsection{Revising typed sequences}

This process consists of revising which types in a case base contribute to the improvement of the CBR planning algorithms. The refinemen of domain types is relevant to the CBR cycle because sequences of certain types can be meaningless. For example, in transportation domains such as Logistics, objects being transported can generate sequences that store knowledge like \{Load-in-Vehicle, no-ops, Unloadfrom-Vehicle $\}$. This information clearly stores the process of moving packages. However, the domain type for places (location) can generate sequences with transitions that do not hold anything relevant in the problem. Package locations are represented with predicate $a t$, in the form (at object place). Thus, the typed sequences generated for the object place will only keep different occurrences of the $a t_{2}$ property, and these type of sequences do not necessarily contribute to a right advice during the replay process.

Accordingly, we have designed a refinemen process that evaluates how a domain case base performs in comparison to the same case base but removing sequences of specifi types. Figure 9 shows the pseudo-code that determines the best set of types for a domain given a validation set of problems $V$ and the planning algorithm $\alpha$ (i.e., one of the CBR variations presented in the previous section). The function evaluate_set runs the problems in $V$ with the algorithm $\alpha$ using the sequences of types in $T_{j}$. The function score gives the score to $T_{j}$ types using the performance time metric used in the IPC-2008, explained in the next section. The RevisingTypes algorithm reproduces a hill-climbing algorithm using score as the evaluation function. The algorithm ends when the best set of types is equal to the set selected in the previous iteration. ${ }^{1}$

\footnotetext{
${ }^{1}$ As the evaluation metric used in IPC-2008 takes into account the performance of other planners/configuration (relative scoring), the best set of types of an iteration is included in the candidates of the next iteration.
}

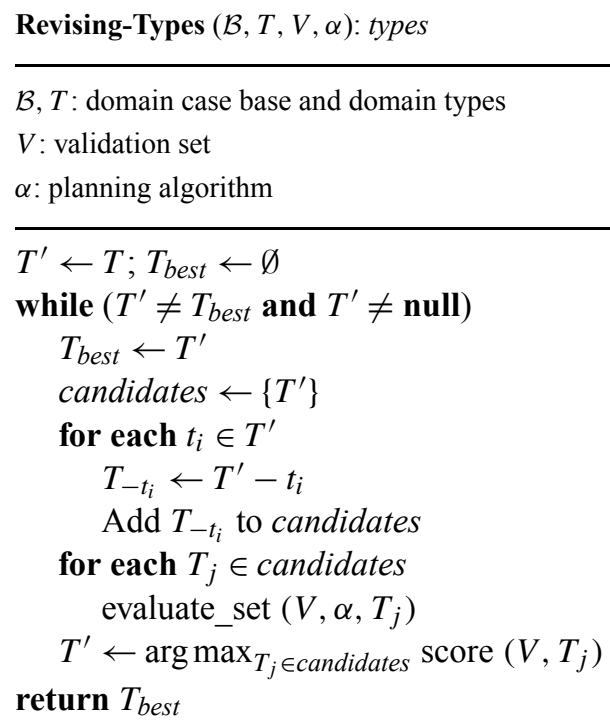

Fig. 9 The algorithm for refinin domain types

Finally the case base $B$ is updated by removing all sequences that belong to types not present in the outcome of the revising-types function.

\section{Experimental evaluation}

This section presents the experiments we have done to evaluate the case-based approach to heuristic planning. We have evaluated this approach in eight STRIPS benchmarks. Two of them (Matching Blocksworld and Parking) were used in the IPC-2008 Learning Track. The rest of them (Blocksworld, Logistics, Depots, Mystery', Satellite, Rovers) are benchmarks commonly used in the planning community. These six domains belong to f ve different domain classes according to Hoffmann's domain topology [14], ${ }^{2}$ which guarantees a reasonable domain variety for evaluation. All domains have random problem generators available in IPCs webs. These generators were used to create different problem sets during training and test phases.

\subsection{Training phase}

For each domain we generated a training set of 20 problems using the random problem generators. These problems are of small size so that it is possible to fin a good quality plan in reasonable time. Each problem was solved with EHC and then, the solution was refine with a Depth-First Branch and Bound (DFBnB) using a time bound of 60 seconds. The best-cost solution found by DFBnB was used to generate the

\footnotetext{
${ }^{2}$ The Matching Blocksworld and Parking domains have not been classifie in the domain topology because [14] was published prior to the IPC 2008 Learning Track.
} 
typed sequences in order to populate the domain case base. The time for generating typed sequences is negligible with respect to the time bound (60 s) for solving each problem. The computational cost of generating a typed sequence is linear in the plan length, and the number of sequences is bounded by the number of objects. We have also generated a validation set of 20 problems for type refinement After a case base was generated with the training set, the revisingtypes algorithm was executed to obtain the best set of types. Then, we removed from the case base all typed sequences belonging to a type not present in the set of best types.

\subsection{Evaluation metrics}

For each domain we have computed the number of solved problems. We have also evaluated the different planning algorithms with the metrics used in the IPC-2008 Learning Track. These metrics measure planning performance in terms of quality (plan length) and CPU Time. Final scores in both metrics are the sum of points given to each problem in a particular domain. For the quality metric, a planner configuratio receives $N_{i}^{*} / N_{i}$ points, where $N_{i}^{*}$ is the minimum number of actions in any solution returned by a planner for the problem $i$, and $N_{i}$ is the number of actions returned by the evaluated configuratio for the problem $i$. If the evaluated planner does not solve the problem, it receives 0 points for this problem. The score for a problem is in the range of $[0,1]$. Likewise, the time metric is computed giving a planner configuratio $T_{i}^{*} / T_{i}$ points, where $T_{i}^{*}$ is the minimum time used by any planner for solving the problem $i$, and $T_{i}$ is the time used by the evaluated planner for solving the problem $i$. The planner receives 0 points if it does not solve the problem.

\subsection{Test phase set up}

For the Matching Blocksworld and Parking domains we have used the 30-problem sets from the target distribution given to the competitors. These distributions have the same difficult as the sets used in the competition. For the rest of the domains, we have built a test set of 30 problems of incremental size, using the random problem generators. We have not used the competition sets because, on the one hand, they have a different number of problems, which makes the comparison with the current metric unfair. On the other hand, the improvements in planning techniques and hardware processing speed have made those sets less attractive for evaluation purposes. For instance, the set used for the Logistics domain in 2000 had 41 goals as the hardest problem. The set used in this evaluation has a maximum of 120 goals.

For the evaluation we used a time bound of 900 seconds for each problem, as did the IPC 2008 Learning Track. The whole experiment was run in an Intel Core 2 Quad CPU with $3 \mathrm{~Gb}$ of memory-bound for the process. All tested configu rations share the same parsing and pre-processing functions, as well as the relaxed plan heuristic. Therefore, the real difference comes from the algorithms. The planning algorithms tested in the evaluation were:

- HC: The hill-climbing algorithm with the helpful actions pruning and a chronological backtracking when the search process runs out of candidates.

- CBR-HC: The CBR Hill-climbing algorithm, implemented with the same features of $\mathrm{HC}$.

- EHC The Enforced Hill-climbing algorithm with the helpful actions pruning. ${ }^{3}$

- CBR-EHC The CBR-EHC algorithm implemented with the same features of EHC.

- WBFS The Weighted Best-First Search without helpful actions pruning. It uses a $w_{h}=3$. Bonet and Geffner [3] reported that $w_{h}$ values between 2 and 10 do not produce significan differences.

- CBR-WBFS The CBR-WBFS algorithm implemented with the same features of WBFS.

\subsection{Results}

Table 1 presents the number of solved problems in the eight evaluation domains for all tested configurations The best

\footnotetext{
${ }^{3}$ This planning configuratio differs from FF search algorithm, because we do not activate the WBFS when EHC fails. This allows us to recognize in which circumstances $\mathrm{EHC}$ is performing well alone.
}

Table 1 Solved problems for the evaluated planning configuration

\begin{tabular}{lrrrcrr}
\hline Domains & HC & CBR-HC & EHC & CBR-EHC & WBFS & CBR-WBFS \\
\hline Blocksworld & 17 & $\mathbf{1 8}$ & 9 & 8 & 8 & 9 \\
Matching-bw & 0 & 0 & 0 & 0 & $\mathbf{6}$ & $\mathbf{6}$ \\
Parking & 21 & $\mathbf{2 5}$ & 12 & 12 & 7 & 7 \\
Logistics & 16 & 21 & $\mathbf{2 6}$ & 25 & 7 & 8 \\
Mystery' & 9 & 20 & 25 & $\mathbf{2 7}$ & 22 & 23 \\
Depots & 25 & 27 & $\mathbf{2 9}$ & $\mathbf{2 9}$ & 21 & 22 \\
Satellite & 27 & $\mathbf{2 9}$ & 19 & 22 & 10 & 9 \\
Rovers & $\mathbf{2 8}$ & 27 & 22 & 27 & 9 & 8 \\
Total & 143 & $\mathbf{1 6 7}$ & 142 & 150 & 90 & 92 \\
\hline
\end{tabular}


Table 2 Scores for CPU Time metric

Table 3 Scores for quality metric

\begin{tabular}{lcccccc}
\hline Domains & HC & CBR-HC & EHC & CBR-EHC & WBFS & CBR-WBFS \\
\hline Blocksworld & 10.21 & $\mathbf{1 4 . 4 1}$ & 1.34 & 2.24 & 2.33 & 2.53 \\
Matching-bw & 0.0 & 0.0 & 0.0 & 0.0 & 3.25 & $\mathbf{6 . 0 0}$ \\
Parking & 14.64 & $\mathbf{2 3 . 5 9}$ & 1.15 & 4.17 & 0.53 & 0.51 \\
Logistics & 2.30 & 12.58 & $\mathbf{1 9 . 4 5}$ & 17.77 & 0.25 & 0.40 \\
Mystery' & 4.34 & 17.91 & 10.74 & $\mathbf{2 1 . 6 4}$ & 2.41 & 2.84 \\
Depots & 12.04 & 18.41 & 17.97 & $\mathbf{2 2 . 3 2}$ & 2.55 & 3.16 \\
Satellite & 10.69 & $\mathbf{2 5 . 7 5}$ & 8.76 & 16.58 & 0.22 & 0.17 \\
Rovers & 18.93 & $\mathbf{2 6 . 5 5}$ & 10.32 & 21.07 & 0.37 & 0.27 \\
Total & 73.15 & $\mathbf{1 3 9 . 2 0}$ & 69.73 & 105.79 & 11.91 & 15.88 \\
\hline
\end{tabular}

\begin{tabular}{lrrrrrr}
\hline Domains & HC & CBR-HC & EHC & CBR-EHC & WBFS & CBR-WBFS \\
\hline Blocksworld & 6.31 & 8.48 & 7.46 & 6.60 & 7.64 & $\mathbf{8 . 5 3}$ \\
Matching-bw & 0.00 & 0.00 & 0.00 & 0.00 & 5.77 & $\mathbf{6 . 0 0}$ \\
Parking & 13.12 & $\mathbf{1 5 . 6 7}$ & 10.09 & 10.48 & 6.76 & 6.29 \\
Logistics & 4.94 & 10.47 & $\mathbf{2 5 . 4 9}$ & 20.82 & 7.73 & 8.80 \\
Mystery' & 6.97 & 16.10 & 20.86 & $\mathbf{2 5 . 7 2}$ & 20.91 & 20.84 \\
Depots & 7.56 & 7.96 & 25.60 & $\mathbf{2 6 . 8 6}$ & 20.31 & 21.09 \\
Satellite & 21.87 & $\mathbf{2 2 . 7 3}$ & 16.91 & 20.97 & 9.79 & 8.78 \\
Rovers & 22.21 & 21.99 & 21.39 & $\mathbf{2 6 . 2 2}$ & 8.88 & 7.81 \\
Total & 83.28 & 103.83 & 127.68 & $\mathbf{1 4 1 . 1 8}$ & 86.86 & 87.13 \\
\hline
\end{tabular}

result per row is presented in bold. The last row shows the sum of solved problems for each configuration CBR$\mathrm{HC}$ was the algorithm that solved the most problems. Note also that each CBR algorithm solved more problems than its standard version. CBR-HC solved 24 more problem than $\mathrm{HC}$, which shows the benefi of the CBR pruning strategy. CBR-EHC solved 8 more problems than EHC, revealing a discrete improvement when using the ordering strategy in greedy search. However, CBR-WBFS only solved 2 more problems than WBFS, indicating that CBR is more appropriate for greedy search algorithms than for best $\mathrm{f}$ rst techniques.

We give a detailed analysis for each domain further in this section. Table 2 shows the fina scores using the time metric. Each domain can receive from 0 to 30 points. Getting the score of 30 means that the planner solved thirty problems in the test set and no other planner solved any problem in less time. The last row presents the sum of the scores obtained in each domain. CBR-HC was the algorithm that got the top score for the time metric. As in solved problems, each CBR algorithm obtained better results than its normal algorithm, especially for greedy search algorithms (in those algorithms the CBR version got double the score than its corresponding non-learning version). Even when the number of solved problems is the same or fairly similar, CBR-HC and CBREHC could solve the same problems in less time. The main reason for this improvement is the reduction in the number
Table 4 Node evaluation average for problems solved by HillClimbing and CBR Hill-Climbing

\begin{tabular}{lccr}
\hline Domains & Common & HC & CBR-HC \\
\hline Blocksworld & 15 & 3823.5 & $\mathbf{3 2 3 5 . 0}$ \\
Matching-bw & 0 & $*$ & $*$ \\
Parking & 21 & 3270.9 & $\mathbf{2 6 8 1 . 4}$ \\
Logistics & 16 & 11016.4 & $\mathbf{7 1 3 9 . 8}$ \\
Mystery' & 9 & 73412.1 & $\mathbf{3 9 . 7}$ \\
Depots & 25 & $\mathbf{1 8 3 6 . 5}$ & $\mathbf{1 8 5 9 . 5}$ \\
Satellite & 27 & 4039.8 & $\mathbf{1 9 0 2 . 7}$ \\
Rovers & 26 & 1931.0 & $\mathbf{1 3 2 9 . 9}$ \\
\hline
\end{tabular}

of evaluations during the search. Likewise, Table 3 shows the scores using the quality metric. Again, each CBR algorithm improved its standard version. In this case CBR-EHC got the top score. This difference arises, as we will see later in this section, because in some domains $\mathrm{HC}$ and $\mathrm{HC}-\mathrm{CBR}$ obtained plans of poor quality.

Table 4 presents the node evaluation average obtained by $\mathrm{HC}$ and $\mathrm{CBR}-\mathrm{HC}$ in problems solved by both configuration (indicated by the "common" column). CBR-HC evaluated fewer nodes in six out of eight domains on average. We do not show a comparison in the Matching Blocksworld domain, because no configuratio solves any problem. Likewise, Table 5 shows the node evaluation average for EHC and CBR-EHC in problems solved by both configurations 
Table 5 Node evaluation average for problems solved by EHC and CBR-EHC

\begin{tabular}{llll}
\hline Domains & Common & EHC & CBR-EHC \\
\hline Blocksworld & 7 & 3543.1 & $\mathbf{2 1 7 6 . 4}$ \\
Matching-bw & $*$ & $*$ & $*$ \\
Parking & 10 & 10456.3 & $\mathbf{4 8 4 7 . 5}$ \\
Logistics & 12 & $\mathbf{1 5 9 2 . 6}$ & 4643.9 \\
Mystery' & 25 & $\mathbf{1 1 2 0 . 1}$ & 2647.6 \\
Depots & 29 & 3627.4 & $\mathbf{1 5 3 1 . 2}$ \\
Satellite & 19 & 1992.6 & $\mathbf{1 0 5 5 . 1}$ \\
Rovers & 22 & 2629.1 & $\mathbf{1 0 3 6 . 4}$ \\
\hline
\end{tabular}

Table 6 Node evaluation average for problems solved by WBFS and CBR-WBFS

\begin{tabular}{lcrc}
\hline Domains & Common & WBFS & CBR-WBFS \\
\hline Blocksworld & 8 & $\mathbf{2 1 0 4 . 5}$ & 3491.5 \\
Matching-bw & 6 & 20878.2 & $\mathbf{7 7 9 1 . 9}$ \\
Parking & 6 & 11479.3 & $\mathbf{7 0 4 9 . 3}$ \\
Logistics & 7 & 19724.4 & $\mathbf{7 3 9 6 . 3}$ \\
Mystery' & 22 & 16136.5 & $\mathbf{1 2 8 7 2 . 3}$ \\
Depots & 21 & 20676.5 & $\mathbf{1 0 8 9 8 . 7}$ \\
Satellite & 9 & $\mathbf{5 8 5 9 . 1}$ & 8636.9 \\
Rovers & 8 & $\mathbf{5 5 8 5 . 8}$ & 10867.1 \\
\hline
\end{tabular}

In this case, CBR-EHC was better in f ve out of eight domains. Finally, Table 6 shows the node evaluation average for WBFS and CBR-WBFS. Although CBRWBFS was better in $f$ ve domains, we do not consider these results as relevant as before since in many domains the number of commonly solved problems is small when compared to the problem set size. In the following sections we explain the results for each domain separately.

\subsubsection{The Blocksworld domain}

The Blocksworld domain is a well-known domain in automated planning. The tasks consist of configurin towers of blocks and placing them with an arm robot. Although it is one of the oldest benchmarks, it is still considered a challenging one. The domain only has the Block type, therefore the refinemen process does not make any changes. The case base only contains 26 sequences, revealing that from a Block perspective, the set of possible transitions is restricted. Figure 10 shows the possible transitions between different typed sub-states in the domain. For instance, suppose that a block on the table with a block on top (sub-state 5) should finis on top of another block and clear (sub-state 2). To reach this goal, it is necessary that the block becomes clear and then held by the arm, before reaching the fina position. This example matches blocks $B$ and $D$ on the example in Fig. 7. Using this knowledge, one can deduce a set

\begin{tabular}{|c|c|c|}
\hline $\begin{array}{c}2:\left(\text { on }_{1} \text { clear }_{1}\right) \\
\text { 文 } \\
1:\left(\text { on }_{1} o_{2}\right)\end{array}$ & $\Leftrightarrow 3:\left(\right.$ holding $\left._{1}\right) \Leftrightarrow$ & 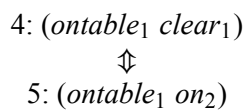 \\
\hline
\end{tabular}

Fig. 10 Possible transitions between typed sub-states in the Blocksworld domain

of invariants for the domain. Nevertheless, typed sequences have the advantage of only keeping the knowledge appearing in a training example. For instance, the typed sequence with transitions (1-2-3-4-3-2) represents a common behavior for a block, which continuously appears in the training examples. By contrast, the sequence with transitions (5-4-3-2$3-4$ ), even though it can be deduced from a domain analysis, never appears in a training example (i.e. in a good quality plan, one does not pick a block up from the table, put it on another block, and then place it back again on the table).

Regarding the results, CBR-HC solved one more problem than $\mathrm{HC}$, but both algorithms produced plans of poor quality. On the other hand, the rest of algorithms solved fewer problems, just with a small improvement on CBRWBFS, which obtains the top score for the quality metric. Although typed sequences seem to capture the right knowledge for this domain, using them at planning time does not offer a significan scalability improvement. First, there is a strong goal dependency in this domain, which can not be handled using an object-perspective approach as ours. Second, CBR algorithms still rely on the heuristic function, which is fairly misleading in this domain. In order to take advantage of the key knowledge stored in Blocksworld sequences it is necessary to complement the search with an additional technique such as a goal agenda or pre-computed landmarks [16].

\subsubsection{The matching Blocksworld domain}

This domain is a variation of the Blocksworld where each block has a polarity, either positive or negative. There are also two arms with polarity. The main difference with the standard Blocksworld is that if a block is held by an arm of wrong polarity, the block becomes damaged and no other block can be placed on the top of it. Blocks get damaged when they are placed by a wrong polarity arm, but not when picked up from the table or unstacked from other block. This fact makes recognizing dead-ends a difficul task. Even though there are alternative representations for this domain, it was designed in this form, to analyze difficultie that arise with the relaxed plan heuristic (i.e., the relaxed task never damages a block, thus both the relaxed plan and the heuristic estimation are wrong).

As in Blocksworld, tasks consist of placing towers of blocks in specifi configurations The refinemen process kept the Hand type and left out the Block type. This does not make much sense because Block sequences capture the 
right knowledge, including the properties block_positive 1 and block_negative ${ }_{1}$ in their corresponding typed sub-states. So, the learned knowledge is useless at planning time because of two reasons. First, Block sequences can not recognize UNSTACK and PICK-UP actions as dangerous, because in those steps blocks do not lose the solid 1 property, the one that indicates the block is not damaged. The block loses this property in a following action if the used arm is of the opposite polarity. The second reason is that the relaxed planning graph does not take into account that blocks can be damaged (this appears in the delete lists, not considered in the computation of the relaxed planning graph). Therefore, the relaxed plan is rather different than the needed one, leaving the set of helpful actions incorrect. Thus, trying to recommend an action from a set of wrong ones is just a waste of time. Even with Hand sequences, the results reveal that algorithms using helpful actions pruning could not solve any problem. WBFS and CBR-WBFS solve the same number of problems, but CBR-WBFS gets top scores for time and quality metrics.

\subsubsection{The parking domain}

This domain comprises a set of curb locations where a set of cars can be parked. Each curb has the restriction of having at most two cars parked. The tasks consist of moving from one configuratio of parked cars to another config uration of parked cars. The domain used in the IPC-2008 Learning Track has a faw. A valid state can lead to an invalid state after applying the action MOVE-CURB-TO-CAR using the same constant for the moved car and the destination car (i.e. the car where the car is moved to). For our tests we used a modifie version of this domain including the precondition (not $(=$ ?car ? cardest) $)$ in the action. Thereby, no invalid states can be reached from a valid one. In the competition, heuristic planners could recognize these invalid states as dead-ends. Nevertheless, the evaluation of these nodes is useless because it is not possible to reach the goals from them. The main consequence of the $\mathrm{faw}$ is the overhead caused by generating and evaluating the invalid states. We preferred to work with the f xed domain, since that way we can give a better interpretation of the scalability improvement achieved by the learning techniques. The refinemen process does not discard any type in this domain. Sequences from both Car and Curb types were used for the test phase. CBR-HC performed very well in this domain, solving four more problems than HC. CBR-HC also got top scores for time and quality metric. Getting 15.67 points in quality with 25 solved problem reveals the algorithm did not get good quality plans. However, this ratio is acceptable when compared with other domains. The rest of the algorithms solved fewer problems, but each CBR algorithm performs better than its standard version. The improvements in the three

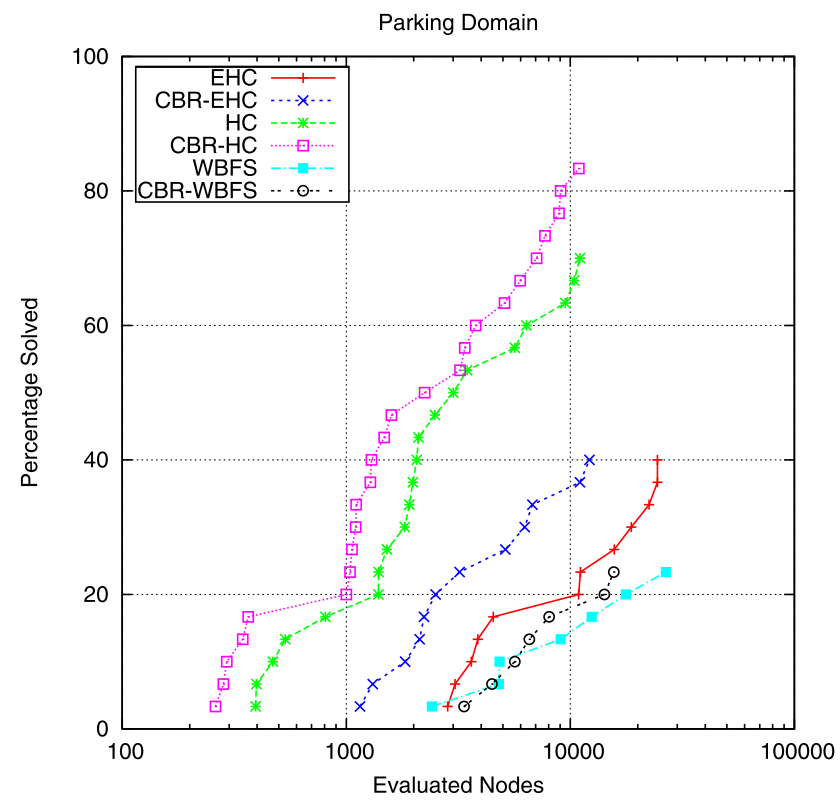

Fig. 11 Percentage of solved problems in increasing evaluated nodes in the Parkingdomain

CBR algorithms are due to a great reduction in the number of evaluated nodes, as can be appreciated in Fig. 11. The $x$-axis (in logarithmic scale) represents the number of evaluated nodes. Each point in the $y$-axis indicates the percentage of solved problems, when evaluating at most $x$ nodes per problem. Thus, left-most lines indicate a better performance.

\subsubsection{The logistics domain}

This transportation domain is considered to be an easy benchmark. The difficult of this domain comes when handling a large number of objects. The tasks comprise moving packages from different locations and different cities using trucks within cities and airplanes among cities. The refine ment process left out the City and Truck types, keeping the Package, Location, Airport and Airplane types. In this case, the relaxed plan footprint is crucial for retrieving the right sequences. Suppose that a package in a location, producing the typed sub-state $\left(a t_{1}\right)$, needs to be in another location in the goal state, which also produces the same typed sub-state. Therefore, in this scenario, the only way of retrieving the correct sequence for the package is identifying the middle transitions it will have. Figure 12 shows all possible combinations of places for delivering a package in Logistics. Wide arrows indicate airplane movement and narrow arrows indicate truck movements. In the list, the combinations 2 and 3 correspond to the same typed sequence. The rest of the combinations produce one sequence each. Different actions for moving vehicles and properties achieved in different RPG layers make footprints distinguishable. Moreover, the Package type has exactly f ve sequences. All of them are retrieved correctly when solving new problems in Logistics. 


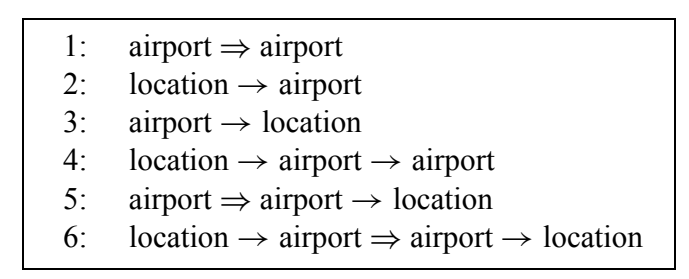

Fig. 12 Different combinations of places for delivering a package in the Logistics domain

Fig. 13 A generic invariant for a cargo in the Mystery' domain

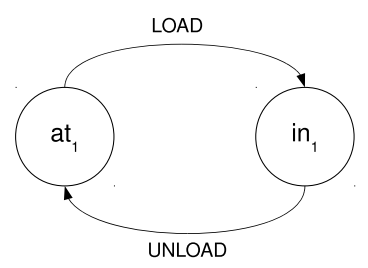

Regarding the results, CBR-HC solved fve more problems than $\mathrm{HC}$, but $\mathrm{EHC}$ got the best performance in terms of solved problems and evaluation metrics. CBR-EHC does not achieve any improvement, because the domain definitio has the operators ordered in the right way such that preferring one successor for evaluation does not benefi the search process. Thus, grounded actions for loading or unloading packages from different vehicles appear before actions for moving vehicles. Actions in the frst group always improve the heuristic value, so there is no need to re-order successors. Additional experiments using a Logistics version with the inverted actions confirme this explanation. In this case, CBR recommendations gave the right advice for sorting actions and therefore for improving EHC.

\subsubsection{The mystery' domain}

This domain is a transportation domain also known as Mprime. The tasks comprise moving objects among different locations using vehicles of limited capacity and having fuel limitation. The refinemen process selected the Cargo type as the only useful one. The other types (i.e., Vehicle, Location, Space, Fuel) were removed. Contrary to what happens in the Logistics domain, in the Mystery' domain all the transported objects have the same sequence. This typed sequence corresponds to the known invariant shown in Fig. 13.

Having this typed sequence only in the replay table forces CBR-HC to directly select action LOAD and UNLOAD whenever they appear as candidates (i.e., no other recommendation could be given). Additionally, actions for moving vehicles (MOVE) and actions for moving fuel (DONATE) are applied taking into account the heuristic estimation. This effect allows the algorithms to safely skip evaluations when actions for loading or unloading a cargo are part of helpful actions. The results show that CBR-HC solved 11 more problems than HC. In general, CBR-HC found plans with less node evaluations than HC. The great difference in the average though, was due to a single problem that CBR-HC solved with 56 evaluations, but $\mathrm{HC}$ evaluated more than 600 thousand nodes and took 412 seconds. Additionally, CBREHC solved 2 more problems than EHC and it got top scores for time and quality metrics.

\subsubsection{The depots domain}

The Depots domain is a combination of the Logistics and Blocksworld domains. The tasks are comprised of trucks transporting crates around depots and distributors. Using hoists, crates must be stacked onto pallets or on top of other crates at their fina destination. As useful types, the refine ment process chooses the set \{Crate, Depot, Hoist, Pallet, Truck\}. The Distributor type was left out. Both Depot and Distributor represent places, so we could think that if one of them is useless, the other is useless too. However, problems normally have more distributors, therefore the refine ment prefer Distributor sequences because they produced more overhead. Depot sequences were not discarded because having them did not degrade the performance, however they did not provide valuable knowledge. Regarding the obtained results, EHC and CBR-EHC solved the same number of problems, but CBR-EHC got a better time score given that it reduces the number of evaluated nodes. It also obtained a slightly better result in the quality score. CBRHC solved two more problems than HC. However, HC and CBR-HC performed poorly in terms of quality, due to goal interaction, which is relevant in this domain, as it is in the Blocksworld domain. WBFS and CBR-WBFS performed reasonably well, considering that they do not prune actions that are not helpful actions (i.e., they have to handle more nodes). CBR-WBFS solves one problem more than WBFS, getting slightly better scores in terms of time and quality.

\subsubsection{The satellite domain}

This domain comprises a set of satellites with different instruments, which can be operated in different modes. The tasks consist of taking images of certain targets in a particular format (mode). The refinemen process does not mark any type as useless. Thus, all domain types (e.g. Satellite, Instrument, Mode and Direction) were used in CBR algorithms. HC-CBR solved more problems than any other algorithm, and it also obtained the best scores for the time and quality metrics. The difficult of a problem relies on the number of objects, but tasks are essentially the same as in simple problems. Accordingly, CBR-HC uses typed sequences most of the time to directly select the next action, producing a great reduction in the number of evaluated nodes. Figure 14 shows the percentage of solved problems when increasing the number of evaluated nodes per problem. We can appreciate that both CBR-HC and CBR-EHC need much fewer nodes than their normal versions in order 


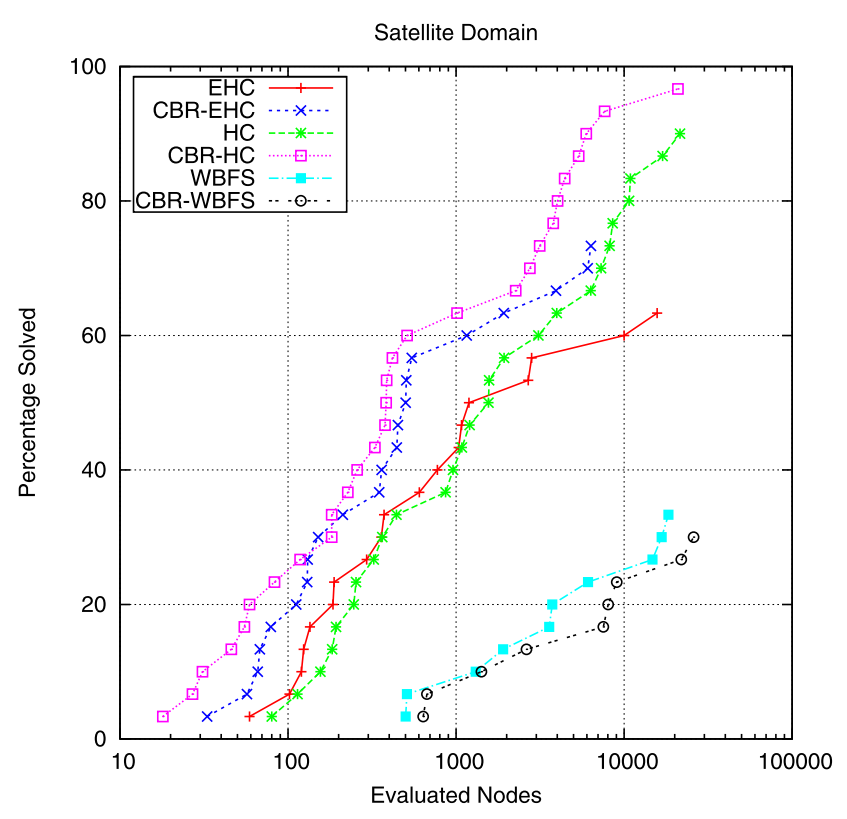

Fig. 14 Percentage of solved problems when increasing evaluated nodes in the Satellite domain

to solve the same percentage of problems. CBR-EHC obtains a good improvement over EHC because the node evaluation order is relevant in this domain. Actions such as CALIBRATE may be evaluated first since it always improves the heuristic value of its parent state. However, the high branching factor makes both WBFS and CBR-WBFS a bad choice for solving problems in this domain. This branching factor is high because a satellite can point to any direction at any time.

\subsubsection{The rovers domain}

This domain is a simplificatio of the tasks performed by the autonomous exploration vehicles sent to Mars. The tasks consist of navigating the rovers, collecting samples of soils and rocks, and taking images of different objectives. The refinemen process left out the Waypoint type and kept the rest of the types: Camera, Lander, Mode Objective, Rover and Store. This is probably because Camera sequences keep the information about taking images, and Store sequences about samples. Thus, Waypoint sequences only produce overhead in the recommendation computations. HC solved one problem more than CBR-HC, but CBR-HC showed a reduction in terms of time. These differences exist because CBRHC could solve many problems faster than HC. CBR-EHC solved f ve more problems than EHC, revealing that the node evaluation order is also important in this domain. The reduction in the number of evaluated nodes (see Table 5) allowed the algorithm to scale better than $\mathrm{EHC}$ and therefore to solve more problems. It also got the top score for the quality metric. As happened in Satellite, WBFS and CBR-WBFS performed significantl worse due to the high branching factor imposed by not using the helpful actions.

\subsubsection{Summary}

The previous analysis shows that rather than having a general rule for predicting the performance of CBR algorithms, the result will depend on the domain representation and the characteristics of its search space. CBR-HC performs well in domains with a big branching factor and many solutions. In these cases, CBR-HC can achieve a big reduction in the number of evaluated nodes. This advantage reduces when problems have strong goal ordering (i.e., there is a fewer number of different solution paths). This weakness arises because retrieved typed sequences are not related to each other, and therefore the CBR algorithms have no information about which sequence is the best one to replay at each state, or which sequences should be replayed at the same time.

On the other hand, EHC-CBR is also able to reduce the number of evaluated nodes in several domains, and it is less affected by the goal ordering issue than HC-CBR. Nevertheless, EHC-CBR partially relies on the heuristic function as EHC does. Therefore, EHC-CBR will solve problems faster than EHC where EHC can lead the search to a solution. However both algorithms will perform poorly with a misleading heuristic. Finally, results showed that WBFS-CBR has no clear advantage over the other algorithms regardless of the evaluated domain. In general, altering $f(n)$ with a CBR recommendation value does not pay off if many nodes are expanded and evaluated anyway. For this reason we think that our approach is more suitable for greedy search algorithms rather than for best-firs ones, such as WBFS.

\section{Related work}

Typed sequences are closely related to the domain invariants deduced with TIM [11]. The f rst versions of several domains written in PDDL (Planning Domain Definitio Language) do not have types in their definition Domain invariants helped to discover implicit types in the domain, which allows a GRAPHPLAN-like planner to instantiate a subset of possible actions, while taking into account the constraints that discovered types imposed. Nowadays, most domains have the object types in their PDDL definition Domain invariants could be used to improve the guidance of a heuristic planner, but to our knowledge it has not been tried yet. On the other hand typed sequences have the advantage that they only code an observed object type transition. A finit state machine define from TIM can encode all valid transitions, but not all of them may be part of a good plan. Additional research is needed to clarify in which domains a deduced invariant or a learned sequence could represent different guidance for a planner.

Our work is also related to OAKPLAN [23], a case-based planner that uses kernel functions to retrieve a set of similar problems with their associated plans. Then, one of these 
plans is adapted to provide a solution. The main difference with our work is that their cases represent the whole planning problem while in our approach we can use information from different problems since we retrieve a case for every single object. Also, they perform case reuse as a local search on the retrieved plan, while we use previous cases to recommend nodes. Previously, other approaches were integrated in learning-based planners. A relevant work is Prodigy/Analogy [25], where lines of reasoning are transferred to the new problem, reproducing/replaying the steps stored in the retrieved cases. Cases in ANALOGY represent the whole planning problem, therefore it is also different to our approach which is object-centered. Many other researchers have applied CBR to planning. The most relevant ones are summarized in [24] and [6]. More recently, CBR has been applied to hierarchical planning such as in HTNMAKER [17]. HTN-Maker learns new methods for decomposing hierarchical tasks. It tries to reduce the engineering effort of designing the domain knowledge for controlling the search, which in the case of hierarchical planning is written within the action model. In our approach, the action model is fi ed and the objective is to modify the search algorithm, so we can use the learned knowledge to guide the search.

Regarding other learning techniques applied to planning, the most representative vision comes from the set of participants of the IPC-2008 Learning Track. The system CABALA used typed sequences to support the generation of lookahead states from a relaxed plan. It performed poorly in the competition because the algorithm (not presented in this work) strongly depends on the quality of the relaxed plan. Other systems such as OBTUSEWEDGE [27], and ROLLER [9], performed better. These systems learn a generalized policy [19], which is a function that maps a metastate to the action that should be applied. When it is hard to induce such a target function, a lazy learning technique such as CBR becomes an alternative for acquiring domaindependent knowledge.

\section{Conclusions}

We have presented in this article an alternative approach to represent and use domain-dependent knowledge for handling scalability issues in domain-independent planning. The technique was developed within the case-based reasoning model, which involves saving, retrieving, using and revising the learned knowledge. We can summarize the contributions of this work as follows:

- Typed sequences: a compact way for abstracting plans and their corresponding state transitions from an objectcentered perspective. Typed sequences can retain substate transitions that commonly appear in problems, which seems easier than deducing all possible sub-state transitions from a domain analysis.
- Relaxed plan footprint: a retrieval key that is used to recognize the right sequences when they have differences only in intermediate steps.

- CBR algorithms: Modification to heuristic algorithms that allow us to integrate the knowledge given by typed sequences in a soft fashion, which also takes into account the guidance of the heuristic estimations.

Results on eight planning benchmarks showed the benefit of using typed sequences during the search process. Improvements obtained by different CBR algorithms were due to the reduction in the number of evaluated nodes. Since computing the relaxed plan heuristic is expensive in terms of time, any technique that can alleviate this burden will scale better in large problem instances. Accordingly, typed sequences can be complemented with other techniques that handle node evaluation issues. As future work we are planning to use CBR node recommendations to validate the action policy of ROLLER [9]. The new algorithm will propose a set of candidate actions to be applied using the relational decision trees, and then will select the actions that replay a typed sequence stored in the case base.

In addition, we want to research alternative representations for typed sequences, in order to enrich the information they can encode. As discussed previously, the CBR algorithms have no information of which sequence to replay firs or which sequences should be replayed at the same time. We want to include information from the causal graph of the planning task [13] to handle this limitation. We also want to enrich typed sequences for handling more complex domains [10].

Finally, the planning community has some interest in synthesizing plans that contain user preferences. This idea is implemented through state preferences and giving rewards or penalties to certain actions. Thus, as future work, we plan to extend our approach by giving type sequences the possibility of acquiring user preference knowledge. As done in CBR algorithms, this information could be used to select or prefer certain actions among the search candidates.

Acknowledgements This work has been partially supported by the Spanish MEC projects PELEA: TIN2008-06701-C03-03 and PlanInteraction: TIN2011-27652-C03-02.

\section{References}

1. Aamodt A, Plaza E (1994) Case-based reasoning: foundational issues, methodological variations, and system approaches. AI Commun 7(1):39-59

2. Blum A, Furst M (1995) Fast planning through planning graph analysis. In: Mellish CS (ed) Proceedings of the 14th international joint conference on artificia intelligence, IJCAI-95, vol 2. Morgan Kaufmann, Montreal, pp 1636-1642

3. Bonet B, Geffner H (2001) Planning as heuristic search. Artif Intell 129(1-2):5-33 
4. Castillo L, Fernández-Olivares J, García-Pérez O, Palao F (2006) Bringing users and planning technology together. Experiences in siadex. In: Proceedings of the 16th international conference on automated planning and scheduling (ICAPS 2006). AAAI Press, Menlo Park

5. Coles A, Fox M, Smith A (2006) A new local search algorithm for forward-chaining planning. In: Proceedings of the 17th international conference on automated planning and scheduling (ICAPS2007), Providence, RI, USA

6. Cox M, Muñoz-Avlia H, Bergmann R (2005) Case-based planning. Knowl Eng Rev 20(3):283-287

7. De la Rosa T, Jiménez S, Borrajo D (2008) Learning relational decision trees for guiding heuristic planning. In: Proceedings of the 18 th international conference on automated planning and scheduling

8. De la Rosa T, Jiménez S, García-Durán R, Fernández F, GarcíaOlaya A, Borrajo D (2009) Three relational learning approaches for lookahead heuristic planning. In: Working notes of ICAPS 2009 workshop on planning and learning, pp 37-44

9. De la Rosa T, Jiménez S, Fuentetaja R, Borrajo D (2011) Scaling up heuristic planning with relational decision trees. J Artif Intell Res 40:767-813

10. Della Penna G, Magazzeni D, Mercorio F (2012) A universal planning system for hybrid domains. Appl Intell 36:932-959

11. Fox M, Long D (1998) The automatic inference of state invariants in TIM. J Artif Intell Res 9:317-371

12. Ghallab M, Nau D, Traverso P (2004) Automated planning, theory and practice. Morgan Kaufmann, San Mateo

13. Helmert M (2006) The fast downward planning system. J Artif Intell Res 26:191-246

14. Hoffmann J (2005) Where "ignoring delete lists" works: local search topology in planning benchmarks. J Artif Intell Res 24:685-758

15. Hoffmann J, Nebel B (2001) The FF planning system: fast plan generation through heuristic search. J Artif Intell Res 14:253-302

16. Hoffmann J, Porteous J, Sebastia L (2004) Ordered landmarks in planning. J Artif Intell Res 22:215-278

17. Hogg C, Muñoz-Avila H, Kuter U (2008) HTN-MAKER: learning htns with minimal additional knowledge engineering required. In: Proceedings of the 20th AAAI conference. AAAI Press, Menlo Park

18. Kuzu M, Cicekli NK (2012) Dynamic planning approach to automated web service composition. Appl Intell 36:1-28

19. Martin M, Geffner H (2004) Learning generalized policies from planning examples using concept languages. Appl Intell 20:9-19

20. McGann C, Py F, Rajan K, Ryan H, Henthorn R (2008) Adaptative control for autonomous underwater vehicles. In: Proceedings of the 23rd AAAI conference. AAAI Press, Menlo Park

21. Nayak P, Kurien J, Dorais G, Millar W, Rajan K, Kanefsky R (1999) Validating the ds-1 remote agent experiment. In: Artificia intelligence, robotics and automation in space

22. Richter S, Westphal M (2010) The LAMA planner: guiding costbased anytime planning with landmarks. J Artif Intell Res 39:127177

23. Serina I (2010) Kernel functions for case-based planning. Artif Intell 174:1369-1406

24. Spalazzi L (2001) A survey on case-based planning. Artif Intell Rev 16:3-36. citeseer.ist.psu.edu/kettler94massively.html

25. Veloso M, Carbonell J (1993) Derivational analogy in PRODIGY: automating case acquisition, storage, and utilization. Mach Learn 10(3):249-278

26. Vidal V (2004) A lookahead strategy for heuristic search planning. In: Proceedings of the fourteenth international conference on automated planning and scheduling, Whistler, British Columbia, Canada, pp 150-160

27. Yoon S, Fern A, Givan R (2008) Learning control knowledge for forward search planning. J Mach Learn Res 9:683-718

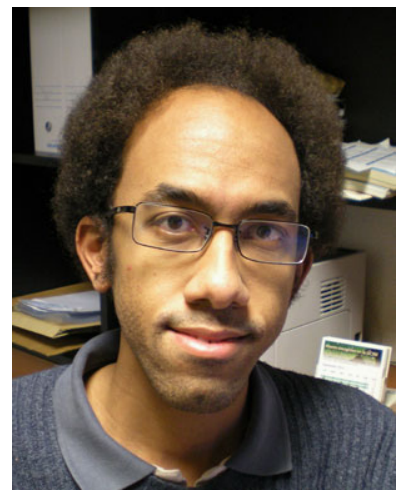

Tomás de la Rosa is a Ph.D. Assistant Professor at Unversidad Carlos III de Madrid (UC3M). He received his Ph.D. in Computer Science from the UC3M in 2010. He is member of the Planning and Learning Group (PLG) at UC3M since 2005. He has published 14 journal and conference papers in the feld of Automated Planning and Machine Learning. He was co-organizer of the Workshop on Planning and Learning in the International Conference of Automated Planning and Scheduling (ICAPS) in 2009. He has participated in 10 research projects funded by European Union and Spanish research council, including 4 projects for transferring knowledge to the industry.

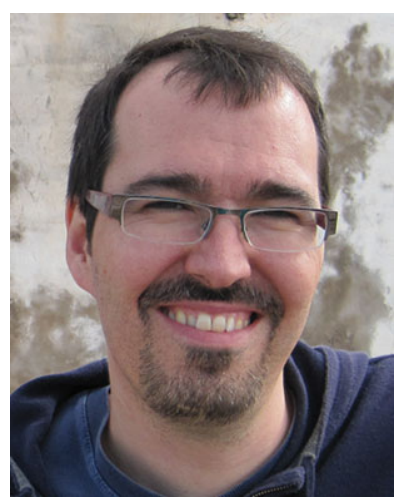

Angel García-Olaya is Associate Professor at Unversidad Carlos III de Madrid (UC3M) since 2004. He received his Ph.D. and B.Sc. in Telecommunications from UPM in 2004 and 1999 respectively. He also has a M.Sc. on Health Informatics from University of Athens, 1997. He is author of about 40 journal and conference papers, in the field of Telemedicine, Automated Planning and Robotics, being awarded with a prize of innovation in Telemedicine by University of Santiago de Compostela in 2004 and with the Best Paper Award in the 2011 Conference of the Spanish Association for Artificia Intelligence. He has participated in 15 research projects funded by European Union and Spanish research council.

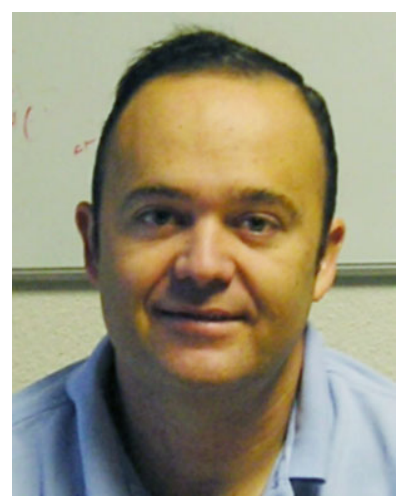

Daniel Borrajo is a University Professor of Computer Science (CS) at Unversidad Carlos III de Madrid (UC3M) since 1998. He received his $\mathrm{Ph} . \mathrm{D}$. in CS from Universidad Politécnica de Madrid (UPM) (1990, doctoral prize), and a B.Sc. in CS (1987) also from UPM. He has been also a pre- and postdoctoral visiting researcher at the CS Department of Carnegie Mellon University (1989, 1993-1994, 2007-2008). He has been vicedean (1996-2000 and 2004-2006) and Head of the Department (2000 2002). He founded the Plannning and Learning Group (PLG) in 2004 from the AI group at UC3M (since 1995). He has published over 160 journal and conference papers mainly in the f eld of machine learning and problem solving (including planning). He has served as the Program co-chair of the International Conference on Automated Planning and Scheduling (ICAPS 2013), Conference co-chair of the International Symposium on Combinatorial Search (SoCS 2011 and 2012), Conference co-chair of ICAPS 2006, and Program Chair of the Spanish Conference on Artificia Intelligence. He has been the coordinator and researcher of more than 15 european, national and regional R\&D projects. 